\title{
Effects of Hip Joint Strengthening Exercises and Lumbopelvic Stabilization Exercises on Balance and Instability of Adults with Functional Ankle Instability
}

\author{
Jae-Ik Lee', Tae-Ho Kim²** \\ ${ }^{1}$ Department of Rehabilitation Science, Graduate School, Daegu University, South Korea \\ ${ }^{2}$ Department of Physical Therapy, Daegu University, South Korea
}

Received April 6, 2021; Revised June 8, 2021; Accepted June 20, 2021

\section{Cite This Paper in the following Citation Styles}

(a): [1] Jae-Ik Lee, Tae-Ho Kim, "Effects of Hip Joint Strengthening Exercises and Lumbopelvic Stabilization Exercises on Balance and Instability of Adults with Functional Ankle Instability," International Journal of Human Movement and Sports Sciences, Vol. 9, No. 4, pp. 757 - 764, 2021. DOI: 10.13189/saj.2021.090421.

(b): Jae-Ik Lee, Tae-Ho Kim (2021). Effects of Hip Joint Strengthening Exercises and Lumbopelvic Stabilization Exercises on Balance and Instability of Adults with Functional Ankle Instability. International Journal of Human Movement and Sports Sciences, 9(4), 757 - 764. DOI: 10.13189/saj.2021.090421.

Copyright $\bigcirc 2021$ by authors, all rights reserved. Authors agree that this article remains permanently open access under the terms of the Creative Commons Attribution License 4.0 International License

\begin{abstract}
In subjects with functional ankle instability, lumbopelvic instability causes instability of the hip and lower extremities. The purpose of this study is to investigate the effect of a lumbopelvic stabilization exercise program on the balance and instability of adults with functional ankle instability. 30 adults with functional ankle instability were assigned to a hip joint strengthening exercise group and a lumbopelvic stabilization exercise group at random. General ankle intervention was used in the hip joint strengthening exercise group, whereas a stabilization exercise using a sling was added in the lumbopelvic stabilization exercise group. The dynamic balance ability, static balance ability, and ankle instability were measured. A Paired-sample t-test was conducted to see the within-group changes in dynamic balance, static balance, and ankle instability scale before and after the experiment. To compare the changes between groups, an independent-sample t-test was conducted. The lumbopelvic stabilization exercise group showed increased the posterior-lateral distance and the scores on foot and ankle ability measures $(p<0.05)$. The average movement distance of the center of pressure, average movement speed, and average movement area 95\% decreased significantly $(\mathrm{p}<0.05)$. The ankle instability instrument score decreased $(\mathrm{p}<0.05)$. The results suggest that existing lumbopelvic stabilization exercises not only help reduce the pain and enhance the function of the hip joint they are also an
\end{abstract}

effective approach for adults with functional ankle instability. Lumbo-pelvic stabilization exercise programs that are tailored to individual characteristics, such as functional level, muscle strength, and duration of ankle injury, reduce the frequency of re-injury and stabilize ankles to improve pain and function.

Keywords Balance, Ankle Instability, Lumbopelvic Stabilization

\section{Introduction}

With the increasing population of people engaging in leisure activities and sports, damage related to sports has also been increasing. Ankle sprains account for $85 \%$ of lateral ankle damages caused by ankle inversion. Among patients who have experienced sprained ankle joints, $10 \%$ to $50 \%$ experience chronic pain and re-injury symptoms, such as chronic ankle sprains [1]. This causes functional instability of the ankle and leads to damage occurring even in daily life. These individuals experience weakness and pain in related tissues; for instance, some cannot overcome the situation and easily experience ankle inversion [2]. Chronic instability of the ankle joint is classified as functional or mechanical instability; 
mechanical instability is due to the relaxation or insufficiency of ligaments, which are static stabilizing structures [3]. According to Freeman [4], patients with functional instability complain of joint instability due to incomplete recovery of impaired proprioception during acute ankle sprains. Functional ankle instability reflects subjective instability, and the patient complains of instability. Related factors include impaired muscle power and proprioception [5].

Problems with functional ankle instability include weakening of the calf muscles on the outside of the ankle, decreased proprioception around the ankle joint [6], and a decrease in the ability to control the eccentric contraction of the ankle joint plantarflexion [7].

Proprioceptive neuromuscular control exercises focused on plantarflexion and instep flexion [8], ankle muscle strengthening exercises focused on the ankle joints, functional exercises related to agility [9], Kinesio taping [10], and increased activity of the long calf muscle and inner soleus muscle during treadmill walking using an auditory biofeedback input device [11] have been reported to be effective treatment methods for ankle instability.

Researchers have also investigated changes in body function other than that of the ankle joint. Previous studies have reported changes in hip joint muscle electromyography and strength in patients with chronic ankle instability [12].

Recently, research on hip joint muscles has become more important for the prevention and treatment of injury of the lower extremities [13]. In cases of ankle dysfunction, identification and treatment of the exact cause are vital, and early treatment is necessary to prevent the recurrence of chronic ankle sprains [14]. These results suggest that continuous interaction between the ankle and hip muscles is necessary to keep up the body's postural balance and center of gravity [15]. They also support the claim that decreased hip joint muscle strength can lead to re-injury of the ankle.

Another characteristic of patients with chronic ankle instability is poor postural control [16]. Reduced postural control ability is related to the occurrence of ankle sprains [17]. This implies that ankle sprains can lead to decreased somatosensory ability and reductions in several related abilities. Decreased postural control is an important risk factor for the development of chronic ankle instability from temporary ankle injury [18]. Proprioception, which plays many roles in controlling and maintaining body posture, decreases with an increase in the degree of damage to the ankle [19]. Plantar pressure measurement approach has been reported as a biomechanical measurement method for postural control deficit; this approach analyzes the overall pressure and weight distribution of the ankle; subtalar joint; and the upper, middle, and lower parts of the foot [20]. Plantar pressure measurement can also be measured by checking the movement of the center of pressure while the subject is standing [21]. The direct cause of musculoskeletal disorders can be determined through an analysis of the sole pressure balance; the pressure of the entire foot and a specific area should be measured and analyzed [22]. In addition, the size and distribution of the foot pressure affect the structural and biomechanical functions of the foot. On this basis, foot pressure analysis is usefully performed to examine pathological gait [23].

Interventions have emerged to improve muscle imbalance and mitigate proprioceptive sensation, pain, and decreased balance ability in patients with chronic ankle instability. Exercises have been developed to strengthen the muscles around the ankle joint and the muscles around the hip. Balance training and proprioception training have also been proposed. The effects of these interventions are related; any change in the foot pressure and the center-of-pressure movement path influences the regulation of postural control ability.

There are many studies on the relationship between the lumbopelvic and hip joints [24-27] and the relationship between ankle and hip instability. However, few studies have revealed the relationship between lumbopelvic and ankle instability [28]. In addition, there is limited research on exercise methods that aim to stabilize the lumbopelvic area to resolve functional ankle instability related to the hip joint.

Therefore, the purpose of this study is to determine whether lumbopelvic stabilization exercises affect the balance and instability scale scores of individuals when such methods are combined with existing functional ankle instability improvement exercises.

\section{Method}

\subsection{Subjects}

The subjects of this study were 30 adults with functional ankle instability. They included students enrolled in D University, in Daegu, and ordinary persons belonging to an athletic club. Those who had an ankle instability instrument score of 4 or higher, those with a percentile of $90 \%$ or less on the foot and ankle ability measure, those with persistent anxiety and functional decline in the ankle joint, and those with recurrent ankle joint damage were included as subjects. Those with a history of surgery on the lower extremities, those with balance disorders, those with exercise or treatment experiences that had affected their balance within the last month, and those with paresthesia or muscle paralysis due to neurological disorders were excluded. All subjects were fully informed about the purpose and method of the study before the experiment was conducted, and the experiment was performed after they agreed to voluntarily participate. The general characteristics of the subjects are shown in Table 1 . 
Table 1. The general characteristics of the subjects

\begin{tabular}{cccc}
\hline & STR & STA & P \\
\hline Age(year) & $30.93 \pm 5.44$ & $31.13 \pm 2.89$ & .100 \\
Body height $(\mathrm{cm})$ & $171.86 \pm 5.74$ & $170.60 \pm 7.02$ & .755 \\
Body weight $(\mathrm{kg})$ & $73.26 \pm 12.20$ & $70.73 \pm 14.15$ & .748 \\
BMI index & $24.71 \pm 3.32$ & $24.13 \pm 3.68$ & .612 \\
\hline
\end{tabular}

$\mathrm{SD} \pm$ standard deviation

STR: exercise program group performing hip joint strengthening exercises.

STA: exercise program group conducting lumbopelvic stabilization exercises.

\subsection{Assessment}

The star excursion balance test (SEBT) was used to measure the change in dynamic balance ability before and after exercise. This evaluation predicts the range of motion, proprioception, and muscle strength of the weight-bearing. Because leg muscles and nerves are efficiently controlled so that the axis of movement and balance can be maintained at the base of support in the single foot support posture [29-30]. It helps to do. Gribble [31] reported that this scale showed high validity and test-retest reliability. (ICC $=0.96$ ). In a previous study, instead of the traditional SEBT, a test that measures a total of three directions (anterior, posterior medial, and posterior-lateral) was adopted to evaluate the dynamic balance ability [32]. The average value was obtained from a total of three measurements [33].

A one-leg standing test was performed on a Wii Balance Board (Nintendo ${ }^{\circledR}$ Wii, Nintendo, Japan) with a built-in Bluetooth function to measure the changes in the subjects' static balance before and after exercise (Fig. 2). A balance ability analysis program (Balancia v1.0, Minto System, Seoul, Republic of Korea) was used to quantify the measured values. The Wii Balance Board measured 45 $\mathrm{cm} \times 26.5 \mathrm{~cm}$ and was equipped with four load cells. Data were sampled at $50 \mathrm{~Hz}$ and $12 \mathrm{~Hz}$, and low-pass filtering was performed. In the measurement of balance ability, each subject stood on one foot on the balance measurement device. The position was maintained for 5 seconds, after which the raised leg was brought down. The movement distance, speed, and area 95\% values for the $X$ and $\mathrm{Y}$ axes of the weight center were measured through the analysis program. Center-of-pressure movement distance is the total distance the pressure center point moved during the evaluation time. Center-of-pressure movement speed is the speed at which the pressure center point moved per unit time. Center-of-pressure movement area $95 \%$ is movement area excluding 5\% after making the movement distance into the circle area.

An ankle instability instrument was used to evaluate the ankle instability before and after exercise. This tool consists of nine items and three detailed items, and the subject answered "yes" or "no". The more questions are answered with "yes," the more severe the functional ankle instability; an individual who answers "yes" to more than four questions are determined to have functional ankle instability. Docherty et al. [34] reported that this scale showed high validity and test-retest reliability (ICC $=$ 0.95).

The foot and ankle ability measure was used to evaluate the ankle instability before and after exercise. This scale consists of 21 questions, and each unit is scored on a scale of 4 to 0 , where $4=$ not difficult, $3=$ slightly difficult, $2=$ a little difficult, $1=$ very difficult, and $0=$ impossible. The higher the score, the closer the ankle function is to normal, and lower scores indicate ankle instability. An individual who scores 75.6 points $(90 \%$ of the total score of 84 points) or less is deemed to be having problems in daily life due to ankle instability. Martin et al. [35] reported that this scale showed high validity and test-retest reliability $(\mathrm{ICC}=0.89)$.

\subsection{Experimental Procedure}

Fifteen patients were assigned to the hip joint strengthening exercise group and the lumbopelvic stabilization exercise group at random. The exercises were performed with the aid of a physiotherapist with more than five years of clinical experience. Each group exercise was the same throughout the four weeks: 5 minutes of warm-up exercise, 30 minutes of main exercise (5 minutes for each exercise), and 5 minutes of finishing exercise. Each program was administered for 40 minutes per day, three times a week. The static balance, dynamic balance, and instability scale scores of the subjects were measured before and after the intervention.

The exercise program of the hip joint strengthening exercise group was developed by referring to methods in previous studies [36-38]. The warm-up exercise involved walking on a treadmill for 5 minutes before the main exercise. The main exercise consisted of a total of six exercises; four of these were identical for both groups, and the two remaining exercises were differentiated. As for the hip joint strengthening exercise, the hip joint extension exercise was performed in the prone position, and the hip joint abduction exercise was performed in the side-lying position (Fig. 1).
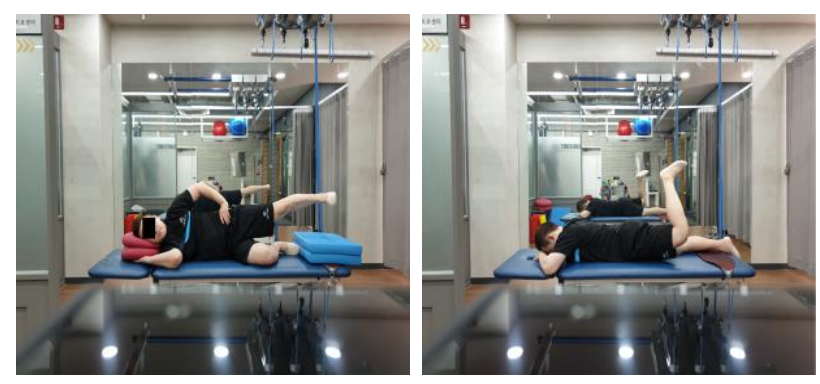

Figure 1. Hip joint strengthening exercise

These movements were performed in three sets consisting of 15 reps each. After each set, the rest period 
was 30 seconds. The difficulty was increased every week through an elastic band (Hygenic $®$, USA) by each subject's condition. For the finishing exercise, stretching and breathing were performed.

The lumbopelvic stabilization exercise was performed in a sling. The difficulty of the lumbopelvic stabilization was set according to individual's ability so that there would be no pain during the exercise; an elastic cord was used to reduce the load on the pelvis. The subjects were instructed to keep up the neutral position of the spine throughout the exercise. The sling exercise was performed via supine pelvic lift and side-lying hip abduction, as suggested in the Neurac method of Kirkesola [39] (Fig. 2). This movement was performed in three sets consisting of 15 reps each.
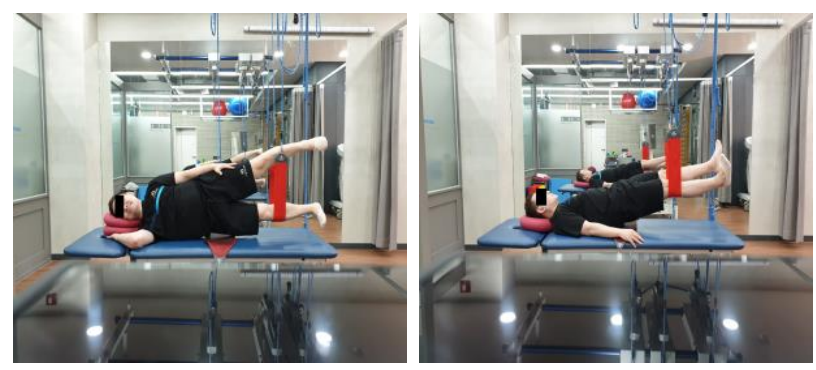

Figure 2. Lumbopelvic Stabilization exercise

The exercise program of the lumbopelvic stabilization exercise group was developed by referring to methods in previous studies. The warm-up exercise involved walking on a treadmill for 5 minutes before the main exercise. The main exercise consisted of a total of six exercises; four of these were identical for both groups, and the two remaining exercises were differentiated. The degree of difficulty was increased every week by changing the motion by each subject's condition. For the finishing exercise, simple stretching and deep breathing were performed. After each set, the rest period was 30 seconds.

\subsection{Statistical Analysis}

The data measured in this study were analyzed using the Statistical Package for the Social Sciences (SPSS) version 20.0 for Windows (SPSS Inc., Chicago). The Kolmogorov-Smirnov test was used for a test of normality. The results of the two groups were compared, and the statistical significance of the difference was determined by performing an independent t-test. A paired t-test was performed to compare the within-group differences between the before- and after-exercise results. The significance level was set as 0.05 .

\section{Results}

After the four weeks of intervention, the anterior distance, posterior medial distance, posterior-lateral distance, and foot and ankle ability measure scores of the hip joint strengthening exercise group significantly increased, and the ankle instability instrument score decreased significantly $(\mathrm{p}<0.05)$. The anterior distance, posterior medial distance, posterior-lateral distance, and foot and ankle capacity scores of the lumbopelvic stabilization exercise group were significantly increased. The center-of-pressure movement distance, center-of-pressure movement speed, center-of-pressure movement area and ankle instability instrument scores were significantly reduced $(\mathrm{p}<0.05)$.

Table 2. The comparison of Star Excursion Balance Test

\begin{tabular}{|c|c|c|c|c|c|c|}
\hline & & Pre & Post & Difference value & $\mathbf{t}$ & $\mathbf{p}$ \\
\hline \multirow{3}{*}{$\begin{array}{l}\text { Ant } \\
(\mathrm{cm})\end{array}$} & STR & $56.84 \pm 4.71$ & $62.88 \pm 5.26$ & $6.04 \pm 4.96$ & 4.715 & $.000^{*}$ \\
\hline & STA & $58.82 \pm 9.37$ & $63.98 \pm 7.80$ & $5.17 \pm 6.16$ & 3.293 & $.005^{*}$ \\
\hline & $\mathrm{t}$ & & & 0.425 & & \\
\hline \multirow{5}{*}{$\begin{array}{l}\text { Post- } \\
\text { Med } \\
(\mathrm{cm})\end{array}$} & $\mathrm{p}$ & & & .674 & & \\
\hline & STR & $78.42 \pm 8.44$ & $86.86 \pm 9.38$ & $8.84 \pm 7.61$ & 6.997 & $.000^{*}$ \\
\hline & STA & $73.51 \pm 11.99$ & $88.02 \pm 12.75$ & $14.51 \pm 11.07$ & 5.404 & $.000^{*}$ \\
\hline & $\mathrm{t}$ & & & -1.607 & & \\
\hline & $\mathrm{p}$ & & & .119 & & \\
\hline \multirow{4}{*}{$\begin{array}{l}\text { Post- } \\
\text { Lat } \\
(\mathrm{cm})\end{array}$} & STR & $82.40 \pm 8.55$ & $89.29 \pm 6.27$ & $6.88 \pm 3.96$ & 6.729 & $.000^{*}$ \\
\hline & STA & $78.00 \pm 14.55$ & $91.38 \pm 12.37$ & $13.44 \pm 9.84$ & 5.297 & $.000^{*}$ \\
\hline & $\mathrm{t}$ & & & -2.391 & & \\
\hline & $\mathrm{p}$ & & & $.024^{*}$ & & \\
\hline
\end{tabular}

$\mathrm{SD} \pm$ standard deviation

STR: Hip joint strengthening exercise group

STA: Lumbopelvic stabilization exercise group 
Table 3. The comparison of one leg standing

\begin{tabular}{|c|c|c|c|c|c|c|}
\hline & & Pre & Post & Difference Value & $\mathbf{t}$ & $\mathbf{p}$ \\
\hline \multirow{4}{*}{$\begin{array}{c}\text { COP } \\
\text { Movement } \\
\text { Distance } \\
(\mathrm{cm})\end{array}$} & STR & $5.61 \pm 1.46$ & $5.14 \pm 1.7$ & $-0.46 \pm 1.98$ & 0.901 & .383 \\
\hline & STA & $7.82 \pm 1.60$ & $2.37 \pm 0.97$ & $-5.44 \pm 1.98$ & 10.634 & $.000^{*}$ \\
\hline & $\mathrm{t}$ & & & 6.873 & & \\
\hline & $\mathrm{p}$ & & & $.000^{*}$ & & \\
\hline \multirow{4}{*}{$\begin{array}{c}\text { COP } \\
\text { Movement } \\
\text { Speed } \\
(\mathrm{cm} / \mathrm{s})\end{array}$} & STR & $4.82 \pm 1.11$ & $4.43 \pm 0.65$ & $-0.38 \pm 1.14$ & 1.302 & .214 \\
\hline & STA & $5.80 \pm 1.30$ & $3.60 \pm 1.70$ & $-2.19 \pm 1.76$ & 4.817 & $.000^{*}$ \\
\hline & $\mathrm{t}$ & & & 3.320 & & \\
\hline & $\mathrm{p}$ & & & $.003^{*}$ & & \\
\hline \multirow{4}{*}{$\begin{array}{c}\text { COP } \\
\text { Movement } \\
\text { Area 95\% } \\
\left(\mathrm{cm}^{2}\right)\end{array}$} & STR & $8.41 \pm 5.69$ & $9.19 \pm 4.91$ & $0.77 \pm 3.52$ & -0.852 & .409 \\
\hline & STA & $12.38 \pm 6.82$ & $4.52 \pm 2.82$ & $-7.85 \pm 4.94$ & 6.156 & $.000^{*}$ \\
\hline & $\mathrm{t}$ & & & 5.504 & & \\
\hline & $\mathrm{p}$ & & & $.000^{*}$ & & \\
\hline
\end{tabular}

$\mathrm{SD} \pm$ standard deviation

STR: Hip joint strengthening exercise group

STA: Lumbopelvic stabilization exercise group

Table 4. The comparison of Ankle instability scale

\begin{tabular}{|c|c|c|c|c|c|c|}
\hline & & Pre & Post & Difference Value & $\mathbf{t}$ & $\mathbf{p}$ \\
\hline \multirow{4}{*}{$\begin{array}{l}\text { FAAM } \\
\text { (Score) }\end{array}$} & STR & $68.46 \pm 5.92$ & $70.93 \pm 3.47$ & $2.46 \pm 3.22$ & -2.961 & $.010^{*}$ \\
\hline & STA & $69.00 \pm 5.71$ & $80.06 \pm 2.84$ & $11.06 \pm 5.83$ & -7.834 & $.000^{*}$ \\
\hline & $\mathrm{t}$ & & & -4.993 & & \\
\hline & $\mathrm{T}$ & & & $.000^{*}$ & & \\
\hline \multirow{4}{*}{$\begin{array}{c}\text { AII } \\
\text { (Score) }\end{array}$} & STR & $5.13 \pm 1.59$ & $4.26 \pm 1.27$ & $-0.86 \pm 0.83$ & 4.026 & $.001^{*}$ \\
\hline & STA & $6.13 \pm 1.55$ & $2.93 \pm 1.16$ & $-3.20 \pm 1.01$ & 12.220 & $.000^{*}$ \\
\hline & $\mathrm{t}$ & & & 6.883 & & \\
\hline & $\mathrm{p}$ & & & $.000^{*}$ & & \\
\hline
\end{tabular}

FAAM: Foot and Ankle Ability Measure

AII: Ankle Instability Instrument

STR: Hip joint strengthening exercise group

STA: Lumbopelvic stabilization exercise group

\section{Discussion}

The purpose of this study is to compare the dynamic balance, static balance, and instability scale scores of subjects with functional ankle instability who performed hip joint strengthening and lumbopelvic stabilization exercises for four weeks.

The SEBT results obtained before and after the intragroup intervention in both exercise groups showed that the anterior, posterior medial, and posterior-lateral distances were significantly increased after the intervention. In the comparison between the groups, only the difference in the posterior-lateral distance of the lumbopelvic stabilization exercise group increased significantly. The SEBT evaluates the dynamic balance ability of a subject.

This finding is similar to that of a previous study that showed that the range of reach in the SEBT of a functional ankle instability group was significantly improved after a rehabilitation exercise program [40]. In the intragroup comparison, the distance in the three directions was significantly increased in both groups, whereas the posterior-lateral distance of the lumbopelvic stabilization exercise group only increased in the 
intergroup comparison. The exercise program of the lumbopelvic stabilization exercise group may have helped improve the posterior-lateral dynamic balance more than the anterior or posterior-lateral dynamic balance of the body. After the intervention, the center-of-pressure movement distance, center-of-pressure movement speed, and center-of-pressure movement area decreased by $95 \%$ in the lumbopelvic stabilization exercise group. The center-of-pressure movement distance was the distance $(\mathrm{cm})$ that the center of pressure point moved during the evaluation time; a decreased distance indicated a well-maintained balance. The center-of-pressure movement speed was the distance $(\mathrm{cm} / \mathrm{s})$ that the center of pressure moved per unit time; a reduced speed meant a well-maintained balance.

The center-of-pressure movement area $95 \%$ would be reduced if the movement distance was made into the circle area, and the balance was well maintained with the area $\left(\mathrm{cm}^{2}\right)$ excluding $5 \%$. In previous studies, subjects with ankle instability showed that the distance of movement of the center of pressure deviated more outwardly than those of subjects without ankle instability [41]. A similar result was found in a previous study showing that balance training improves the postural control and function of subjects with chronic ankle instability; this work identified a decrease in the center-of-pressure movement speed after balance training [42]. Likewise, a previous study found that the range of the center-of-pressure movement area returned within the normal range after intervention [43]. Other previous studies reported that therapeutic exercise using slings restores and improves the balance ability of patients with chronic low back pain [44].

The lumbopelvic stabilization exercises may have increased the participants' hip joint performance by stabilizing the trunk, thereby enabling them to more smoothly adjust the center-of-pressure point in the one-leg standing test. In particular, in the comparison of the balance ability between the groups, all three values decreased more in the lumbopelvic stabilization exercise group than in the hip joint strengthening exercise group.

The change in the balance ability of the hip joint strengthening exercise group indicated that it is insufficient to increase the balance ability only with the existing ankle and hip joint exercise program training.

The ankle instability scale scores significantly changed in both the hip joint strengthening exercise group and the lumbopelvic stabilization exercise group after the intervention.

The foot and ankle ability measure scores significantly increased, and the ankle instability instrument scores significantly decreased. There were also significant differences between the foot and ankle ability measure scores and the ankle instability scores in the comparison between the two groups.

The foot and ankle ability measure scale is assessment tool for people with musculoskeletal disorders of the legs, ankles, and feet. These findings were similar to those of a previous study where the foot and ankle capacity score increased after intervention [45]. Likewise, a previous study showed that the foot and ankle ability measure score increased after ankle reinforcement and balance exercises [46]. Finally, the lumbopelvic stabilization exercises may have stabilized the ankle by increasing the performance ability of the hip joint through stabilization of the trunk.

There are several limitations in this study. First, it was difficult to recruit adult subjects with functional ankle instability. There were only 30 subjects, so it is difficult to generalize the research results. Second, since the exercise program consisted of various movements, it was challenging to determine which specific training influenced a specific result. Third, the control variables could not be easily controlled for other physical activities because it was difficult to control the subjects' physical activities outside the experimental training. Fourth, because of the short training period (four weeks), the duration of the exercise program was unsatisfactory. Considering these limitations, it is necessary to develop an exercise program that will reduce symptoms of ankle instability and improve the one-step function, which is an essential element for walking and running, through an experiment of an exercise program for subjects with functional ankle instability. In addition, future studies will have to be conducted with larger numbers of subjects. Appropriate stabilization exercise program items tailored to individual characteristics, such as function level, muscle strength, and duration of injury of subjects with ankle instability, should be developed and studied.

Lumbopelvic stabilization exercise programs that are tailored to individual characteristics, such as functional level, muscle strength, and duration of an ankle injury, reduce the frequency of re-injury and stabilize ankles to improve pain and function.

\section{Conclusions}

The purpose of this study was to investigate the effect of a lumbopelvic stabilization exercise program on balance, and instability in adults with functional ankle instability. Balance ability, and foot instability score were measured. According to the results, posterior-lateral distance and foot and ankle ability measure scores increased. The average path, velocity, and area $95 \%$ of the center of pressure significantly decreased, and the ankle instability instrument score decreased. The results suggest that existing lumbopelvic stabilization exercises not only help with pain and function of the hip joint but are also an effective approach for adults with functional ankle instability. Lumbopelvic stabilization exercise programs that are tailored to individual characteristics, such as functional level, muscle strength, and duration of an ankle 
injury, reduce the frequency of re-injury and stabilize ankles to improve pain and function.

\section{REFERENCES}

[1] P. Sperryn, "Sports medicine on the line?," Br J Sports Med, 28(1):3, 1994.

[2] J. Hertel, "Functional instability following lateral ankle sprain," Sports Med, 29(5):361-371, 2000.

[3] D. W. Kim, K. S. Sung, "Chronic Lateral Ankle Instability," Journal of Korean Foot \& Ankle Society, 22(2):55-61, 2018.

[4] M. A. Freeman, M. R. Dean, I. W. Hanham, "The etiology and prevention of functional instability of the foot," $J$ Bone Joint Surg Br, 47(4):678-685, 1965.

[5] C. W. DiGiovanni, A. Brodsky, "Current concepts: lateral ankle instability," Foot Ankle Int, 27(10):854-866, 2006.

[6] T. Willems, E. Witvrouw, J. Verstuyft, P. Vaes, D. De Clercq, "Proprioception and Muscle Strength in Subjects With a History of Ankle Sprains and Chronic Instability," J Athl Train, 37(4):487-493, 2002.

[7] J. Fox, C. L. Docherty, J. Schrader, T. Applegate, "Eccentric plantar-flexor torque deficits in participants with functional ankle instability," J Athl Train, 43(1):51-54, 2008.

[8] C. M. Bleakley, S. R. O'Connor, M. A. Tully, "Effect of accelerated rehabilitation on function after ankle sprain: randomised controlled trial," BMJ, 10;340:c1964, 2010.

[9] C. W. Lin, C. E. Hiller, R. A. de Bie, "Evidence-based treatment for ankle injuries: a clinical perspective," J Man Manip Ther, 18(1):22-28, 2010.

[10] C. de-la-Torre-Domingo, I. M. Alguacil-Diego, F. Molina-Rueda, "Effect of Kinesiology Tape on Measurements of Balance in Subjects With Chronic Ankle Instability: A Randomized Controlled Trial," Arch Phys Med Rehabil, 96(12):2169-2175, 2015.

[11] L. Donovan, M. A. Feger, J. M. Hart, "Effects of an auditory biofeedback device on plantar pressure in patients with chronic ankle instability," Gait Posture, 44:29-36, 2016.

[12] S. Van Deun, F. F. Staes, K. H. Stappaerts,"Relationship of chronic ankle instability to muscle activation patterns during the transition from double-leg to single-leg stance," Am J Sports Med, 35(2):274-281, 2007.

[13] M.K. Dwyer, S. N. Boudreau, C. G. Mattacola, "Comparison of lower extremity kinematics and hip muscle activation during rehabilitation tasks between sexes," J Athl Train, 45(2):181-190, 2010.

[14] J. N. Bernier, D. H. Perrin, "Effect of coordination training on proprioception of the functionally unstable ankle," $J$ Orthop Sports Phys Ther, 27(4):264-275, 1998.

[15] P. O. McKeon, J. Hertel, "Systematic review of postural control and lateral ankle instability, part I: can deficits be detected with instrumented testing," $J$ Athl Train, 43(3):293-304, 2008.

[16] T. A. McGuine, J. J. Greene, T. Best, "Balance as a predictor of ankle injuries in high school basketball players," Clin J Sport Med, 10(4):239-244, 2000.

[17] D. Knapp, S. Y. Lee, L. Chinn, "Differential ability of selected postural-control measures in the prediction of chronic ankle instability status," $J$ Athl Train, 46(3):257-262, 2011.

[18] J. W. Feuerbach, M. D. Grabiner, T. J. Koh, "Effect of an ankle orthosis and ankle ligament anesthesia on ankle joint proprioception, Am J Sports Med, 22(2):223-229, 1994.

[19] S. Angın, N. Ilçin, S. S. Yeşilyaprak, "Prediction of postural sway velocity by foot posture index, foot size and plantar pressure values in unilateral stance," Eklem Hastalik Cerrahisi, 24(3):144-148, 2013.

[20] K. M. Guskiewicz, D. H. Perrin, "Research and clinical applications of assessing balance," J Sport Rehabil, 5(1):45-63, 1996.

[21] F. P. Kendall, E. K. MCCreary, P. G. Provance, M. M. Rodgers, W. A. Romani, Muscles: Testing and function, with posture and pain (Kendall, Muscles), Philadelphia: Lippincott Williams \& Wilkins, 2005.

[22] T. R. Han, N. J. Paik, M. S. Im, "Quantification of the path of center of pressure (COP) using an F-scan in-shoe transducer," Gait Posture, 10(3):248-254, 1999.

[23] G. Gravante, G. Russo, F. Pomara, C. Ridola, "Comparison of ground reaction forces between obese and control young adults during quiet standing on a baropodometric platform," Clin Biomech (Bristol, Avon), 18(8):780-782, 2003.

[24] M. K. Chan MK, K. W. Chow, A. Y. Lai AY, "The effects of therapeutic hip exercise with abdominal core activation on recruitment of the hip muscles," BMC Musculoskelet Disord, 18(1):313, 2017.

[25] S. L. Hoffman, M. B. Johnson, D. Zou, "Effect of classification-specific treatment on lumbopelvic motion during hip rotation in people with low back pain," Man Ther, 16(4):344-350, 2011.

[26] J. A. Smith, J. M. Jr. Popovich, K. Kulig, "The influence of hip strength on lower-limb, pelvis, and trunk kinematics and coordination patterns during walking and hopping in healthy women," J Orthop Sports Phys Ther, 44(7):525-531, 2014.

[27] J. P. van Wingerden, A. Vleeming, H. M. Buyruk, K. Raissadat, "Stabilization of the sacroiliac joint in vivo: verification of muscular contribution to force closure of the pelvis," Eur Spine J, 13(3):199-205, 2004.

[28] W. Lim, "Tensile force transmission from upper trunk to the contralateral lower leg throughout posterior oblique sling system," International journal of Human Movement and

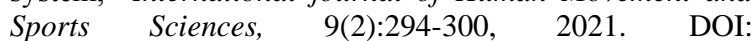
10.13189/saj.2021.090217.

[29] J. E. Earl, J. Hertel, "Lower-extremity muscle activation during the Star Excursion Balance Tests," J Sport Rehabil, 10(2):93-104, 2001. 
[30] A. S. Lengkana, A. A. Rahman, M. N. Alif, G. Mulya, A. Priana, D. B. Hermawan, "Static and dynamic balance learning in primary school students," International journal of Human Movement and Sports Sciences, 8(6):469-476, 2020. DOI: $10.13189 /$ saj.2020.080620.

[31] P. A. Gribble, S. E. Kelly, K. M. Refshauge, C. E. Hiller, "Interrater reliability of the star excursion balance test," $J$ Athl Train, 48(5):621-626, 2013. DOI: $10.13189 /$ saj.2020.080620

[32] J. Hertel J, R. A. Braham, S. A. Hale, "Simplifying the star excursion balance test: analyses of subjects with and without chronic ankle instability," J Orthop Sports Phys Ther, 36(3):131-137, 2006.

[33] K Fullam, B Caulfield, "Kinematic analysis of selected reach directions of the Star Excursion Balance Test compared with the Y-Balance Test," J Sport Rehabil, 23(1):27-35, 2014.

[34] C. L. Docherty, B. M. Gansneder, B. L. Arnold, S. R. Hurwitz. "Development and reliability of the ankle instability instrument," J Athl Train, 41(2):154-158, 2006.

[35] R. L. Martin, J. J. Irrgang, R. G. Burdett, S. F. Conti, "Evidence of validity for the Foot and Ankle Ability Measure (FAAM)," Foot Ankle Int, 26(11):968-983, 2005.

[36] S. A. Hale, J. Hertel, L. C. Olmsted-Kramer, "The effect of a 4-week comprehensive rehabilitation program on postural control and lower extremity function in individuals with chronic ankle instability," J Orthop Sports Phys Ther, 37(6):303-311, 2007.

[37] D. M. Selkowitz, G. J. Beneck, C. M. Powers, "Which exercises target the gluteal muscles while minimizing activation of the tensor fascia lata? Electromyographic assessment using fine-wire electrodes," J Orthop Sports Phys Ther, 43(2):54-64, 2013.

[38] T. Wagner, N. Behnia, W. K. Ancheta, R. Shen, "Strengthening and neuromuscular reeducation of the gluteus maximus in a triathlete with exercise-associated cramping of the hamstrings," J Orthop Sports Phys Ther, 40(2):112-119, 2010.

[39] G. Kirkesola, "Neurac-a new treatment method for long-term musculoskeletal pain," $J$ Fysioterapeuten, 76:16-25, 2009.

[40] S. A. Hale, J. Hertel, L. C. Olmsted-Kramer, "The effect of a 4-week comprehensive rehabilitation program on postural control and lower extremity function in individuals with chronic ankle instability," J Orthop Sports Phys Ther, 37(6):303-311, 2007.

[41] R. M. Koldenhoven, M. A. Feger, J. J. Fraser, "Surface electromyography and plantar pressure during walking in young adults with chronic ankle instability," Knee Surg Sports Traumatol Arthrosc, 24(4):1060-1070, 2016.

[42] P. O. McKeon, J. Hertel, "Systematic review of postural control and lateral ankle instability, part I: can deficits be detected with instrumented testing," $J$ Athl Train, 43(3):293-304, 2008.

[43] N. Matsusaka, S. Yokoyama, T. Tsurusaki, "Effect of ankle disk training combined with tactile stimulation to the leg and foot on functional instability of the ankle," Am J Sports Med, 29(1):25-30, 2001.

[44] J. H. Kim, Y. E. Kim, S. H. Bae, K. Y. Kim, "The effect of the neurac sling exercise on postural balance adjustment and muscular response patterns in chronic low back pain patients," J Phys Ther Sci, 25(8):1015-1019, 2013.

[45] J. L. Schaefer, M. A. Sandrey, "Effects of a 4-week dynamic-balance-training program supplemented with Graston instrument-assisted soft-tissue mobilization for chronic ankle instability," J Sport Rehabil, 21(4):313-326, 2012.

[46] C. J. Burcal CJ, A. Y. Trier, E. A. Wikstrom, "Balance training versus balance training with STARS in patients with chronic ankle instability: A randomized controlled trial," J Sport Rehabil, 26(5):347-357, 2017. 